\title{
Supporting Infotmation
}

\section{Selective Intermolecular C-H Bond Activation - a Straightforward Synthetic Approach to Heteroalkyl Yttrium Complexes Containing Bis(pyrazolyl)methyl Ligand}

Dmitry M. Lyubov, ${ }^{[a]}$ Anton V. Cherkasov, ${ }^{[a]}$ Georgy K. Fukin, ${ }^{[a]}$ and Alexander A. Trifonov $*^{[a],[b]}$

[a] Institute of Organometallic Chemistry of Russian Academy of Sciences Tropinina 49, GSP445, 630950, Nizhny Novgorod (Russia) Fax: 007831 4627497; Tel: 0078314623532 E-mail: trif@iomc.ras.ru

[b] Institute of Organoelement compounds of Russian Academy of Sciences Vavilova str. 28, 119334, Moscow (Russia) 
Table S1. Crystal data and structure refinement for complexes 1, 6-11.

Figure S1. ${ }^{1} \mathrm{H}$ NMR spectrum of $1\left(400 \mathrm{MHz}, \mathrm{C}_{6} \mathrm{D}_{6}, 293 \mathrm{~K}\right)$

Figure S2. ${ }^{13} \mathrm{C}\left\{{ }^{1} \mathrm{H}\right\}$ NMR spectrum of $\mathbf{1}(100 \mathrm{MHz}, 293 \mathrm{~K})$.

Figure S3. ${ }^{1} \mathrm{H}$ NMR spectrum of 6 (400 MHz, $\left.\mathrm{C}_{6} \mathrm{D}_{6}, 293 \mathrm{~K}\right)$.

Figure S4. ${ }^{13} \mathrm{C}\left\{{ }^{1} \mathrm{H}\right\}$ NMR spectra of $6\left(100 \mathrm{MHz}, \mathrm{C}_{6} \mathrm{D}_{6}, 293 \mathrm{~K}\right)$

Figure S5. ${ }^{1} \mathrm{H}$ NMR spectrum of 7 (400 MHz, $\left.\mathrm{C}_{6} \mathrm{D}_{6}, 293 \mathrm{~K}\right)$.

Figure S6. ${ }^{13} \mathrm{C}\left\{{ }^{1} \mathrm{H}\right\}$ NMR spectrum of $7\left(100 \mathrm{MHz}, \mathrm{C}_{6} \mathrm{D}_{6}, 293 \mathrm{~K}\right)$.

Figure S7. ${ }^{1} \mathrm{H}$ NMR spectrum of 8 (400 MHz, $\left.\mathrm{C}_{6} \mathrm{D}_{6}, 293 \mathrm{~K}\right)$.

Figure S8. ${ }^{13} \mathrm{C}\left\{{ }^{1} \mathrm{H}\right\}$ NMR spectrum of $8\left(100 \mathrm{MHz}, \mathrm{C}_{6} \mathrm{D}_{6}, 293 \mathrm{~K}\right)$.

Figure S9. ${ }^{1} \mathrm{H}$ NMR spectrum of $9\left(400 \mathrm{MHz}, \mathrm{C}_{6} \mathrm{D}_{6}, 293 \mathrm{~K}\right)$.

Figure S10. ${ }^{13} \mathrm{C}\left\{{ }^{1} \mathrm{H}\right\}$ NMR spectrum of $9\left(100 \mathrm{MHz}, \mathrm{C}_{6} \mathrm{D}_{6}, 293 \mathrm{~K}\right)$.

Figure S11. ${ }^{1} \mathrm{H}$ NMR spectrum of $\mathbf{1 0}\left(400 \mathrm{MHz}, \mathrm{C}_{6} \mathrm{D}_{6}, 293 \mathrm{~K}\right)$.

Figure S12. ${ }^{13} \mathrm{C}\left\{{ }^{1} \mathrm{H}\right\}$ NMR spectrum of $\mathbf{1 0}\left(100 \mathrm{MHz}, \mathrm{C}_{6} \mathrm{D}_{6}, 293 \mathrm{~K}\right)$.

Figure S13. ${ }^{1} \mathrm{H}$ NMR spectrum of $11\left(400 \mathrm{MHz}, \mathrm{C}_{6} \mathrm{D}_{6}, 293 \mathrm{~K}\right)$.

Figure S14. ${ }^{13} \mathrm{C}\left\{{ }^{1} \mathrm{H}\right\}$ NMR spectrum of $11\left(100 \mathrm{MHz}, \mathrm{C}_{6} \mathrm{D}_{6}, 293 \mathrm{~K}\right)$. 


\begin{tabular}{|c|c|c|c|c|c|c|c|}
\hline & 1 & 6 & 7 & 8 & 9 & 10 & 11 \\
\hline Empirical formula & $\mathrm{C}_{33} \mathrm{H}_{45} \mathrm{~N}_{12} \mathrm{Y}$ & $\mathrm{C}_{44} \mathrm{H}_{63} \mathrm{~N}_{6} \mathrm{OSiY}$ & $\mathrm{C}_{44} \mathrm{H}_{69} \mathrm{~N}_{6} \mathrm{SiY} \cdot\left(\mathrm{C}_{6} \mathrm{H}_{14}\right)$ & $\mathrm{C}_{43} \mathrm{H}_{67} \mathrm{~N}_{6} \mathrm{O}_{2} \mathrm{SiY}$ & $\mathrm{C}_{29} \mathrm{H}_{49} \mathrm{~N}_{4} \mathrm{OSiY}$ & $\mathrm{C}_{72} \mathrm{H}_{90} \mathrm{~N}_{12} \mathrm{Y}_{2} \cdot\left(\mathrm{C}_{4} \mathrm{H}_{8} \mathrm{O}\right)_{2.5}$ & $\mathrm{C}_{52} \mathrm{H}_{70} \mathrm{~N}_{7} \mathrm{OY} \cdot\left(\mathrm{C}_{4} \mathrm{H}_{8} \mathrm{O}\right)_{0.5}$ \\
\hline FW & 698.72 & 809.00 & 885.22 & 817.03 & 586.72 & 1481.64 & 934.11 \\
\hline$T, \mathrm{~K}$ & $100(2)$ & $100(2)$ & $100(2)$ & $100(2)$ & $100(2)$ & $100(2)$ & $100(2)$ \\
\hline Crystal system & Triclinic & Monoclinic & Monoclinic & Monoclinic & Triclinic & Triclinic & Monoclinic \\
\hline Space group & $\mathrm{P}-1$ & $\mathrm{P} 2_{1} / \mathrm{c}$ & $\mathrm{P} 2_{1} / \mathrm{c}$ & $\mathrm{P} 2_{1} / \mathrm{n}$ & P-1 & P-1 & $\mathrm{C} 2 / \mathrm{c}$ \\
\hline $\begin{array}{l}\text { Unit cell } \\
\text { dimensions }\end{array}$ & $\begin{array}{c}a=10.6958(7) \\
b=11.9100(7) \\
c=15.333(1) \\
\alpha=91.9220(1) \\
\beta=96.080(1) \\
\gamma=115.751(1)\end{array}$ & $\begin{array}{c}\mathrm{a}=18.9708(9) \\
\mathrm{b}=11.1377(5) \\
\mathrm{c}=22.706(1) \\
\alpha=90 \\
\beta=113.436(1) \\
\gamma=90\end{array}$ & $\begin{aligned} \mathrm{a}= & 18.1649(9) \\
\mathrm{b}= & 15.8121(8) \\
\mathrm{c}= & 18.5251(9) \\
& \alpha=90 \\
\beta= & 105.805(1) \\
& \gamma=90\end{aligned}$ & $\begin{array}{c}\mathrm{a}=11.2280(2) \\
\mathrm{b}=23.8658(3) \\
\mathrm{c}=16.4287(2) \\
\alpha=90 \\
\beta=92.127(1) \\
\gamma=90\end{array}$ & $\begin{array}{c}\mathrm{a}=10.1876(1) \\
\mathrm{b}=10.8868(1) \\
\mathrm{c}=31.6483(3) \\
\alpha=90 \\
\beta=92.127(1) \\
\gamma=90\end{array}$ & $\begin{array}{c}\mathrm{a}=13.3399(5) \\
\mathrm{b}=13.5884(5) \\
\mathrm{c}=24.700(1) \\
\alpha=78.055(1) \\
\beta=83.095(1) \\
\gamma=62.848(1)\end{array}$ & $\begin{array}{c}\mathrm{a}=46.031(3) \\
\mathrm{b}=10.6303(6) \\
\mathrm{c}=21.541(1) \\
\alpha=90 \\
\beta=104.895(1) \\
\gamma=90\end{array}$ \\
\hline$V, \AA^{3}$ & $1742.4(2)$ & $4401.7(4)$ & $5119.7(4)$ & $4399.3(1)$ & $3146.02(6)$ & $3896.0(3)$ & $10186(1)$ \\
\hline$Z$ & 2 & 2 & 4 & 4 & 4 & 2 & 8 \\
\hline$D_{\text {calc }}, \mathrm{g} / \mathrm{cm}^{3}$ & 1.332 & 1.221 & 1.148 & 1.234 & 1.239 & 1.263 & 1.218 \\
\hline Abs coeff, $\mathrm{mm}^{-1}$ & 1.715 & 1.319 & 1.200 & 1.394 & 1.918 & 1.536 & 1.190 \\
\hline$F(000)$ & 732 & 1720 & 1912 & 1744 & 1248 & 1568 & 3984 \\
\hline Crystal size, $\mathrm{mm}$ & $0.52 \times 0.15 \times 0.11$ & $0.30 \times 0.20 \times 0.13$ & $0.37 \times 0.32 \times 0.32$ & $0.20 \times 0.20 \times 0.10$ & $0.60 \times 0.40 \times 0.30$ & $0.29 \times 0.27 \times 0.14$ & $0.37 \times 0.14 \times 0.14$ \\
\hline $\begin{array}{l}\theta \text { range for data } \\
\text { collection, }\left[{ }^{\circ}\right]\end{array}$ & $1.91-26.00$ & $1.84-26.00$ & $1.17-26.00$ & $1.17-26.00$ & $3.02-27.00$ & $1.71-26.00$ & $1.83-26.00$ \\
\hline Index ranges & $\begin{array}{l}-13 \leq \mathrm{h} \leq 13 \\
-14 \leq \mathrm{k} \leq 14 \\
-18 \leq 1 \leq 18\end{array}$ & $\begin{array}{c}-23 \leq \mathrm{h} \leq 23 \\
-13 \leq \mathrm{k} \leq 13 \\
-27 \leq 1 \leq 28\end{array}$ & $\begin{array}{l}-22 \leq \mathrm{h} \leq 22 \\
-19 \leq \mathrm{k} \leq 19 \\
-22 \leq 1 \leq 22\end{array}$ & $\begin{array}{l}-15 \leq \mathrm{h} \leq 15 \\
-33 \leq \mathrm{k} \leq 33 \\
-23 \leq 1 \leq 23\end{array}$ & $\begin{array}{c}-13 \leq \mathrm{h} \leq 13 \\
-13 \leq \mathrm{k} \leq 13 \\
-40 \leq 1 \leq 40\end{array}$ & $\begin{array}{l}-16 \leq \mathrm{h} \leq 16 \\
-16 \leq \mathrm{k} \leq 16 \\
-30 \leq 1 \leq 30\end{array}$ & $\begin{array}{l}-56 \leq \mathrm{h} \leq 56 \\
-13 \leq \mathrm{k} \leq 13 \\
-26 \leq 1 \leq 26\end{array}$ \\
\hline Reflns collected & 15152 & 37143 & 43171 & 92884 & 52840 & 33941 & 42725 \\
\hline $\begin{array}{l}\text { Independent reflns } \\
R_{\text {int }}\end{array}$ & $\begin{array}{c}6825 \\
0.0153\end{array}$ & $\begin{array}{c}8652 \\
0.0719\end{array}$ & $\begin{array}{l}10048 \\
0.0437\end{array}$ & $\begin{array}{l}12799 \\
0.0896\end{array}$ & $\begin{array}{l}13708 \\
0.0334\end{array}$ & $\begin{array}{l}15263 \\
0.0345\end{array}$ & $\begin{array}{l}10004 \\
0.0901\end{array}$ \\
\hline Completeness to $\theta$ & 99.7 & 100 & 99.8 & 99.8 & 99.8 & 99.7 & 99.9 \\
\hline $\begin{array}{l}\text { Data / restraints / } \\
\text { parameters }\end{array}$ & $6825 / 0 / 439$ & 8652 / 47 / 510 & 10048 / 56 / 591 & $12799 / 4 / 502$ & $13708 / 44 / 704$ & 15263 / $98 / 910$ & $10004 / 30 / 608$ \\
\hline$G O F$ on $F^{2}$ & 1.043 & 0.982 & 1.073 & 1.022 & 1.022 & 1.078 & 0.975 \\
\hline $\begin{array}{l}\text { Final } R \text { indices } \\
{[I>2 \sigma(I)]}\end{array}$ & $\begin{array}{c}R_{1}=0.0262 \\
w R_{2}=0.0681\end{array}$ & $\begin{array}{l}R_{1}=0.0423 \\
w R_{2}=0.0893\end{array}$ & $\begin{array}{l}R_{1}=0.0557 \\
w R_{2}=0.1545\end{array}$ & $\begin{array}{l}R_{1}=0.0549 \\
w R_{2}=0.1068\end{array}$ & $\begin{array}{l}R_{1}=0.0410 \\
w R_{2}=0.0871\end{array}$ & $\begin{array}{l}R_{1}=0.0603 \\
w R_{2}=0.1665\end{array}$ & $\begin{array}{l}R_{1}=0.0533 \\
w R_{2}=0.1131\end{array}$ \\
\hline$R$ indices (all data) & $\begin{array}{c}R_{1}=0.0293 \\
w R_{2}=0.0694\end{array}$ & $\begin{array}{c}R_{1}=0.0765 \\
w R_{2}=0.0974\end{array}$ & $\begin{array}{c}R_{1}=0.0864 \\
w R_{2}=0.1688\end{array}$ & $\begin{array}{c}R_{1}=0.0801 \\
w R_{2}=0.1142\end{array}$ & $\begin{array}{c}R_{1}=0.0511 \\
w R_{2}=0.0906\end{array}$ & $\begin{array}{c}R_{1}=0.0856 \\
w R_{2}=0.1791\end{array}$ & $\begin{array}{c}R_{1}=0.1026 \\
w R_{2}=0.1265\end{array}$ \\
\hline $\begin{array}{l}\text { Largest diff peak } \\
\text { and hole, }\left[\mathrm{e} \AA^{3}\right]\end{array}$ & 0.366 and -0.270 & 0.436 and -0.386 & 1.427 and -0.550 & 1.258 and -1.116 & 0.978 and -1.602 & 2.565 and -2.156 & 0.999 and -0.406 \\
\hline
\end{tabular}

Table S1. Crystal data and structure refinement for complexes 1, 6-11. 


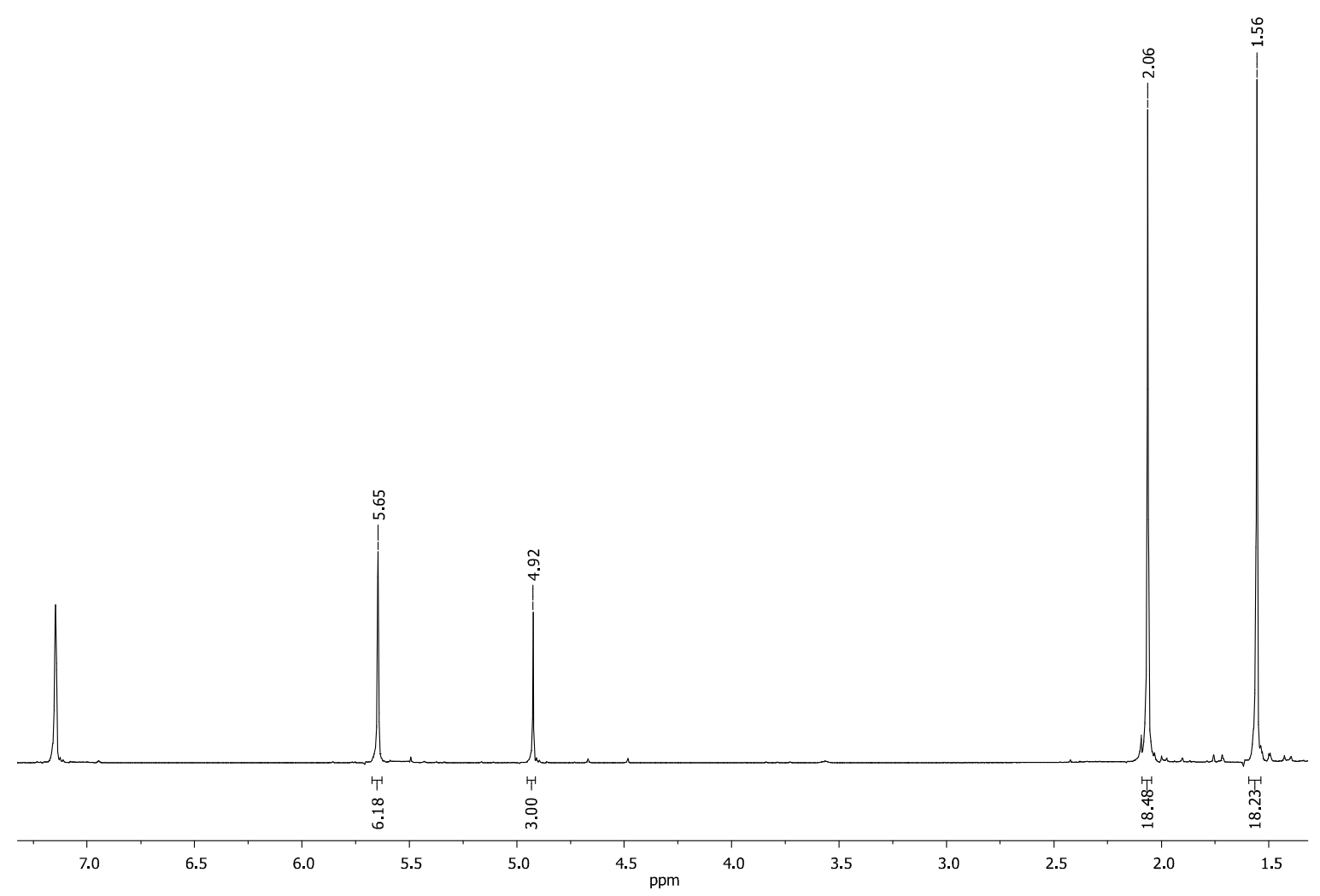

Figure S1. ${ }^{1} \mathrm{H}$ NMR spectrum of 1 (400 MHz, $\left.\mathrm{C}_{6} \mathrm{D}_{6}, 293 \mathrm{~K}\right)$

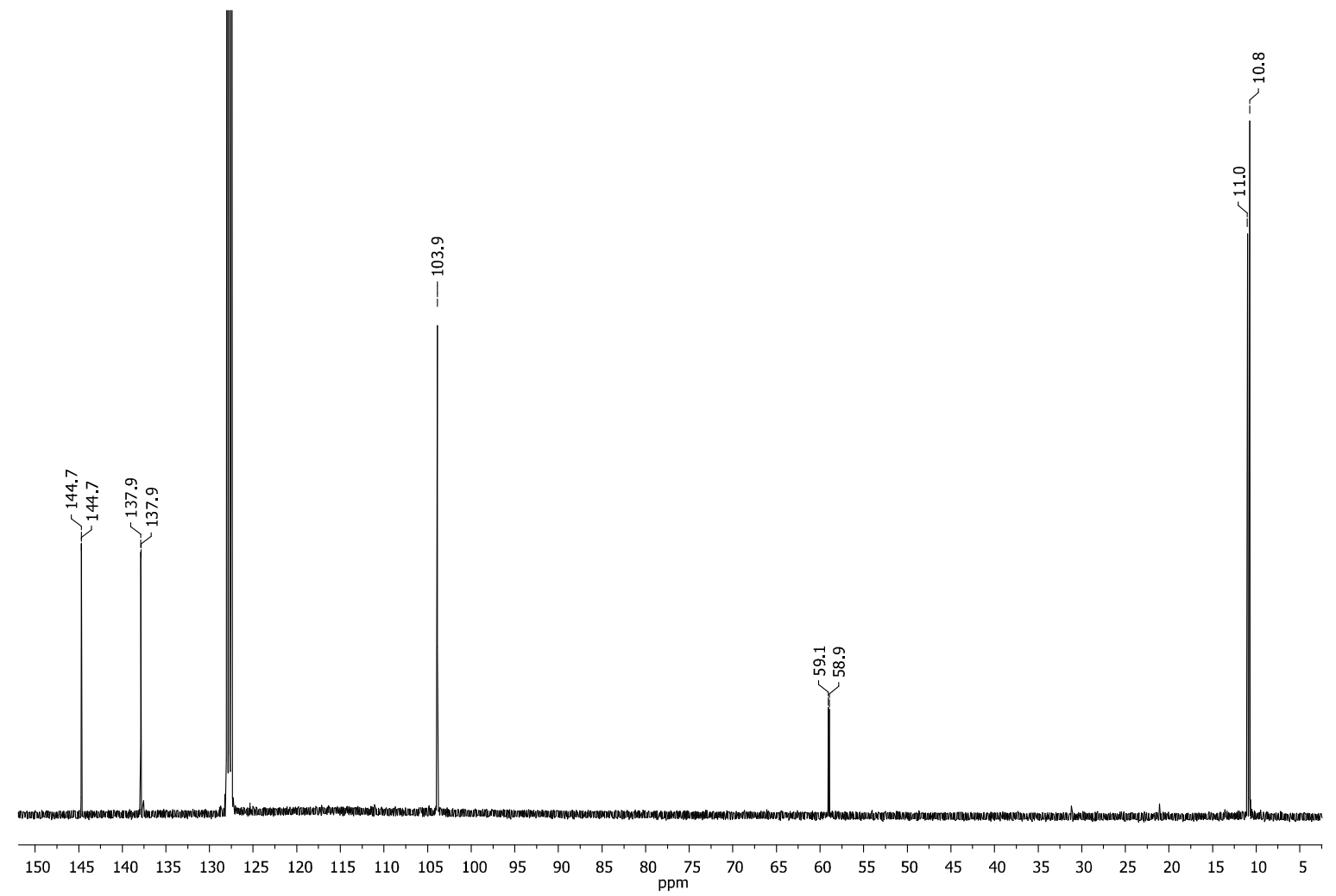

Figure S2. ${ }^{13} \mathrm{C}\left\{{ }^{1} \mathrm{H}\right\}$ NMR spectrum of $\mathbf{1}(100 \mathrm{MHz}, 293 \mathrm{~K})$. 


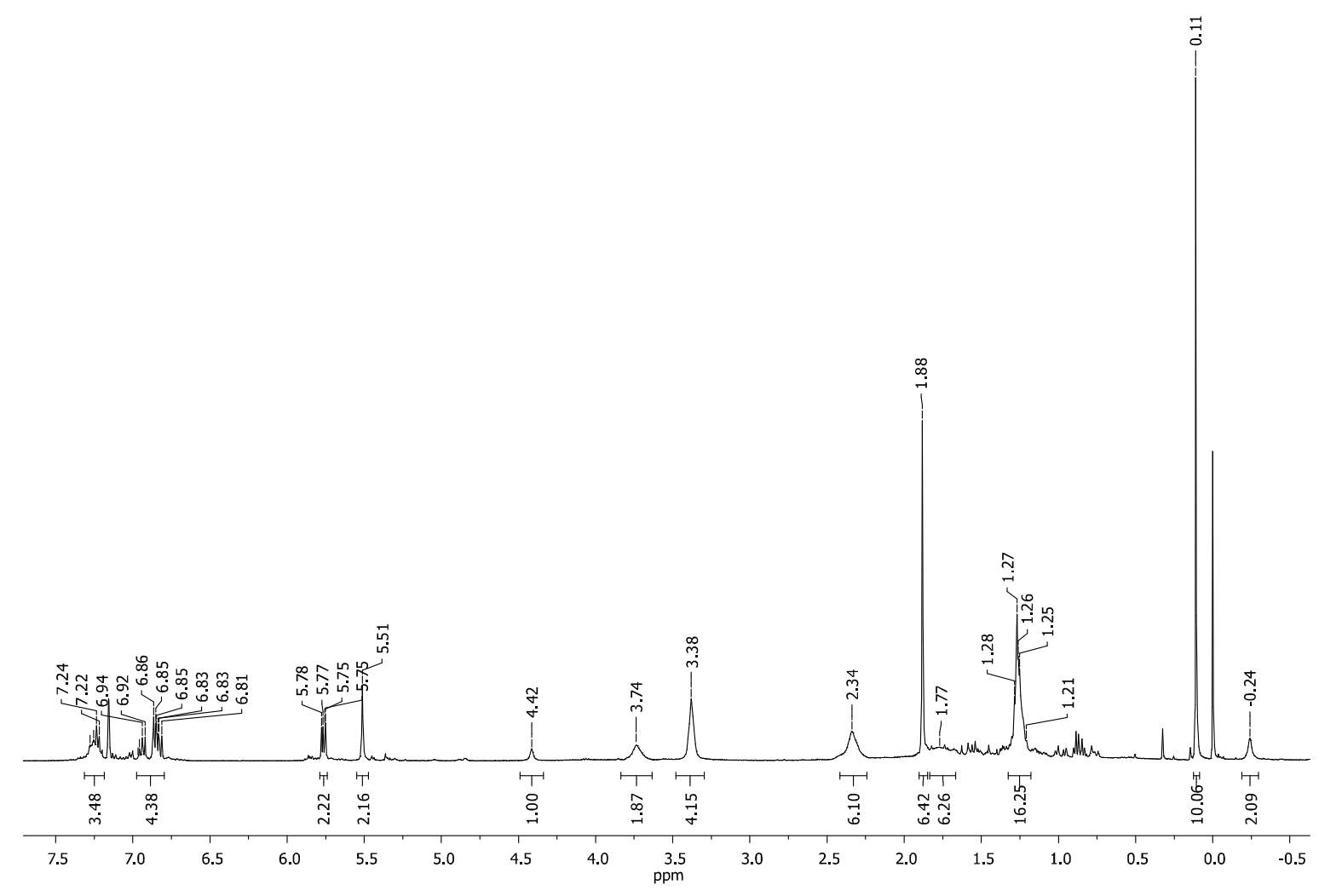

Figure S3. ${ }^{1} \mathrm{H}$ NMR spectrum of 6 (400 MHz, $\mathrm{C}_{6} \mathrm{D}_{6}, 293 \mathrm{~K}$ ).

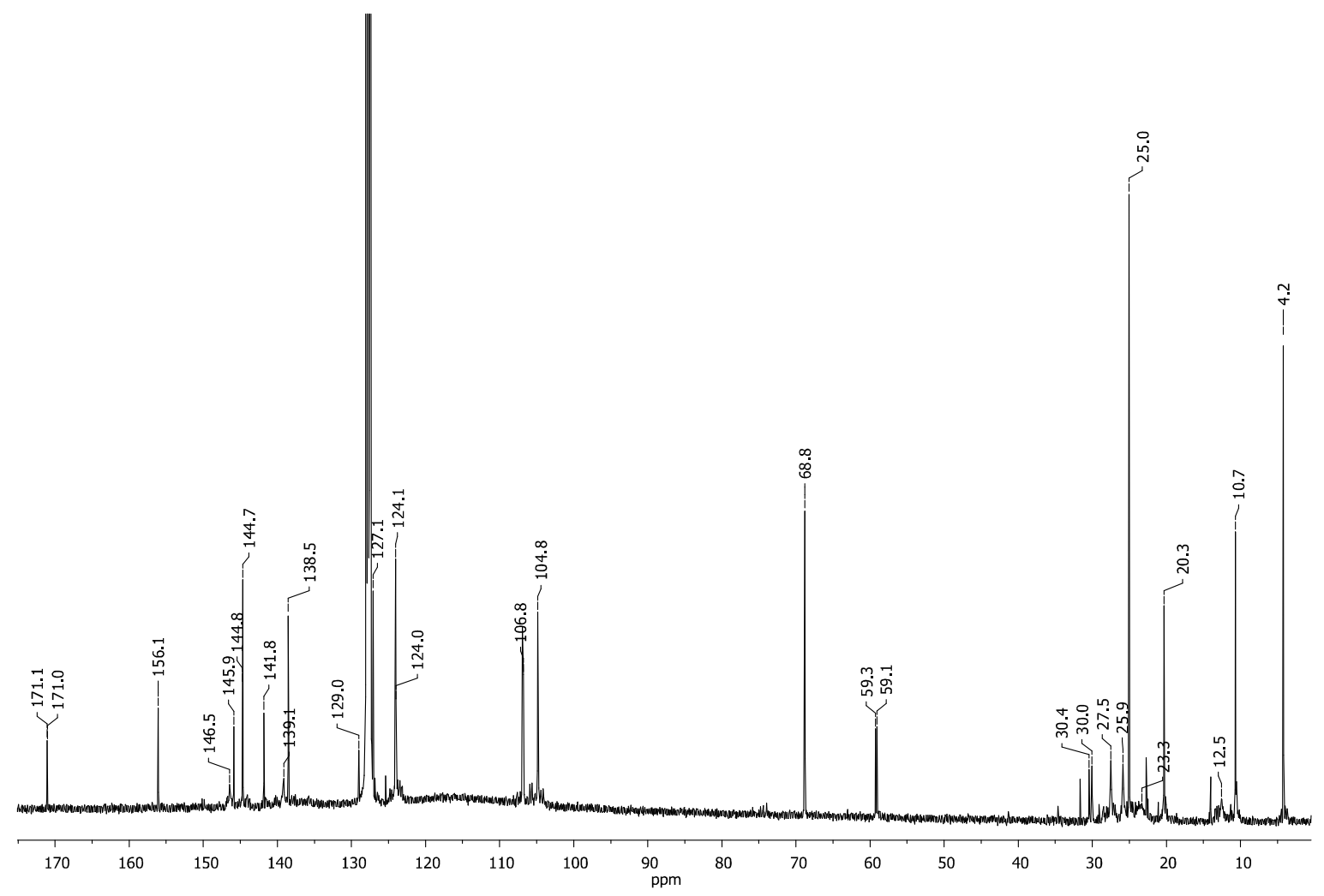

Figure S4. ${ }^{13} \mathrm{C}\left\{{ }^{1} \mathrm{H}\right\}$ NMR spectra of $6\left(100 \mathrm{MHz}, \mathrm{C}_{6} \mathrm{D}_{6}, 293 \mathrm{~K}\right)$ 


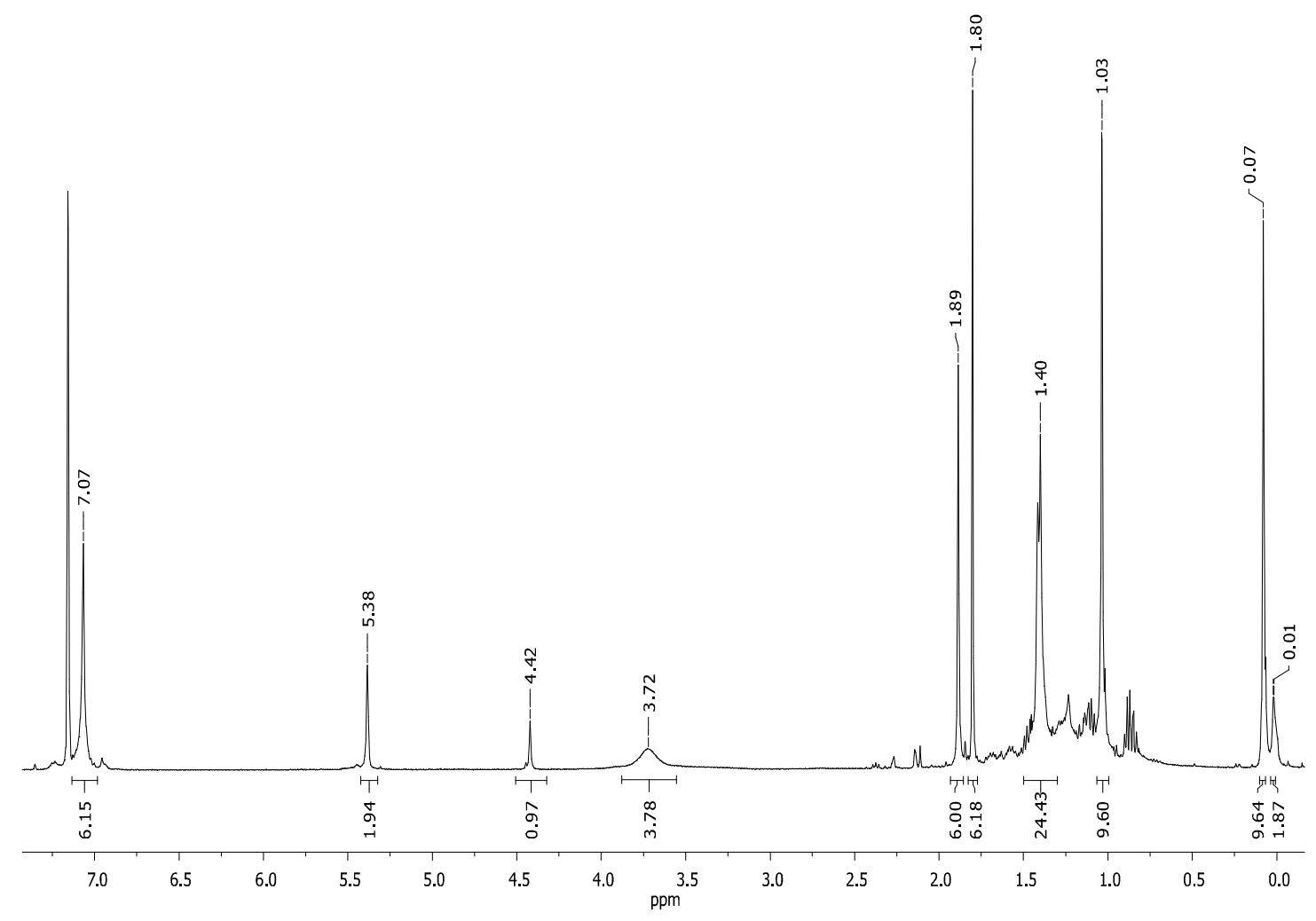

Figure S5. ${ }^{1} \mathrm{H}$ NMR spectrum of 7 (400 MHz, $\left.\mathrm{C}_{6} \mathrm{D}_{6}, 293 \mathrm{~K}\right)$.

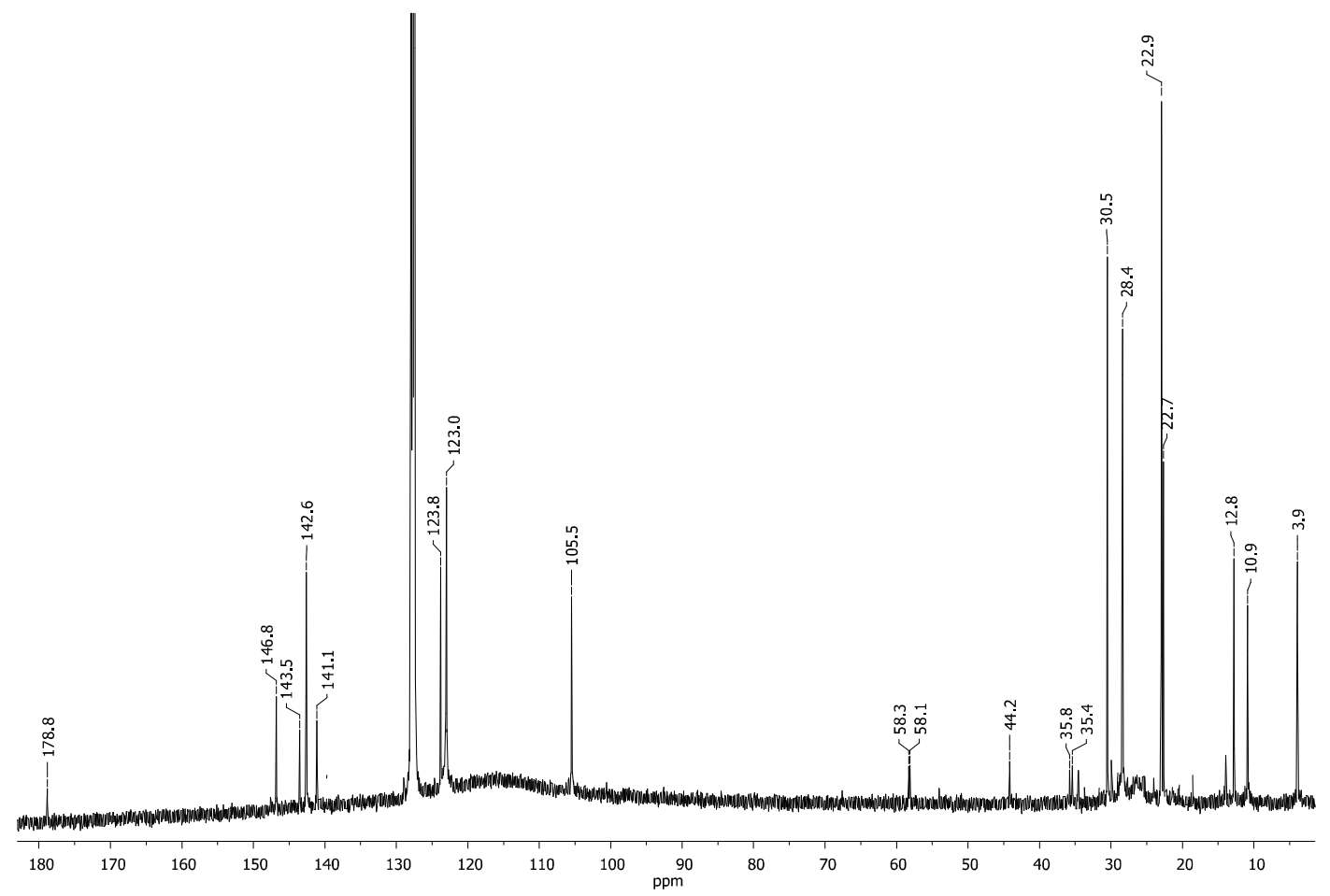

Figure S6. ${ }^{13} \mathrm{C}\left\{{ }^{1} \mathrm{H}\right\}$ NMR spectrum of $7\left(100 \mathrm{MHz}, \mathrm{C}_{6} \mathrm{D}_{6}, 293 \mathrm{~K}\right)$. 


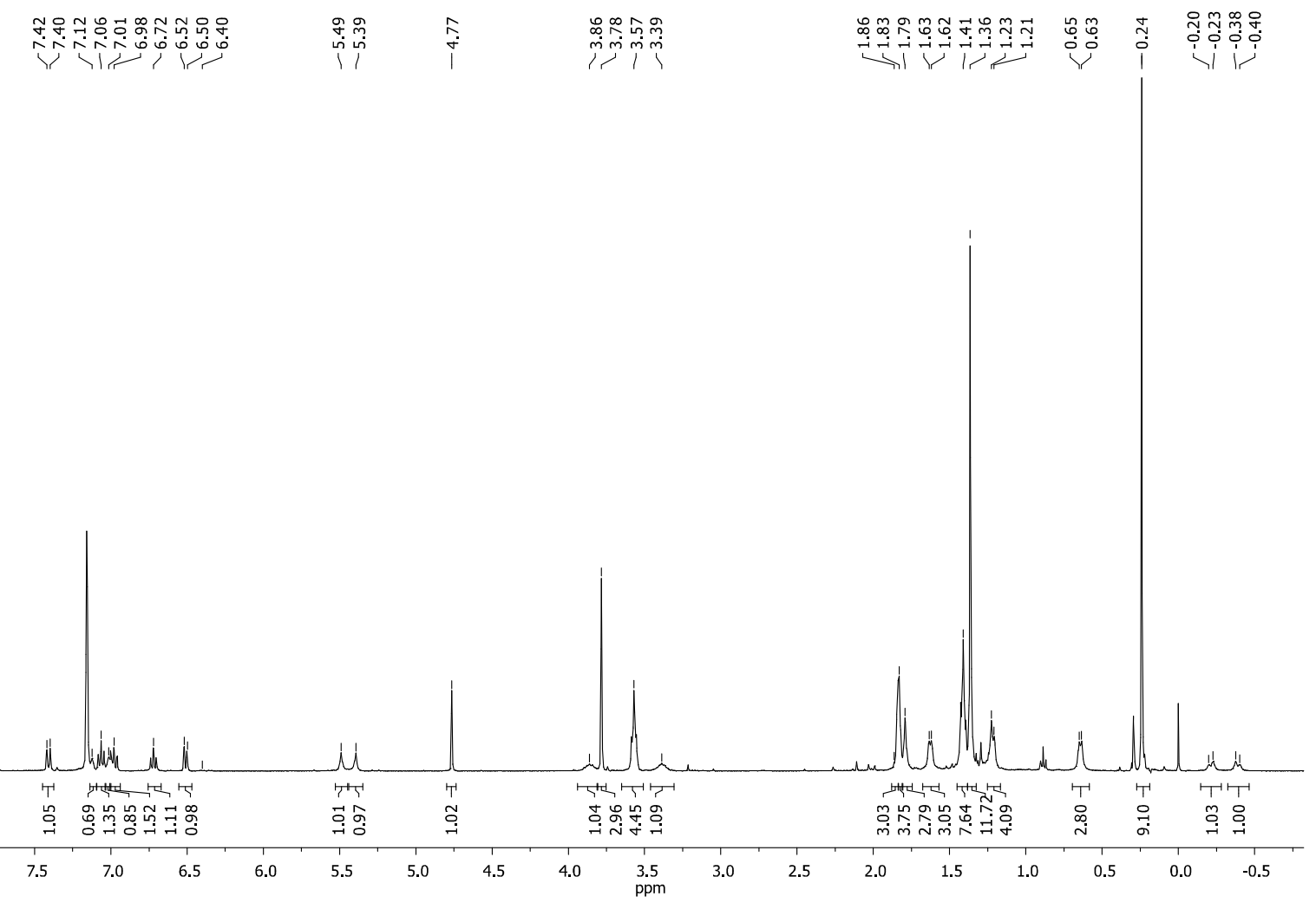

Figure S7. ${ }^{1} \mathrm{H}$ NMR spectrum of $8\left(400 \mathrm{MHz}, \mathrm{C}_{6} \mathrm{D}_{6}, 293 \mathrm{~K}\right)$.

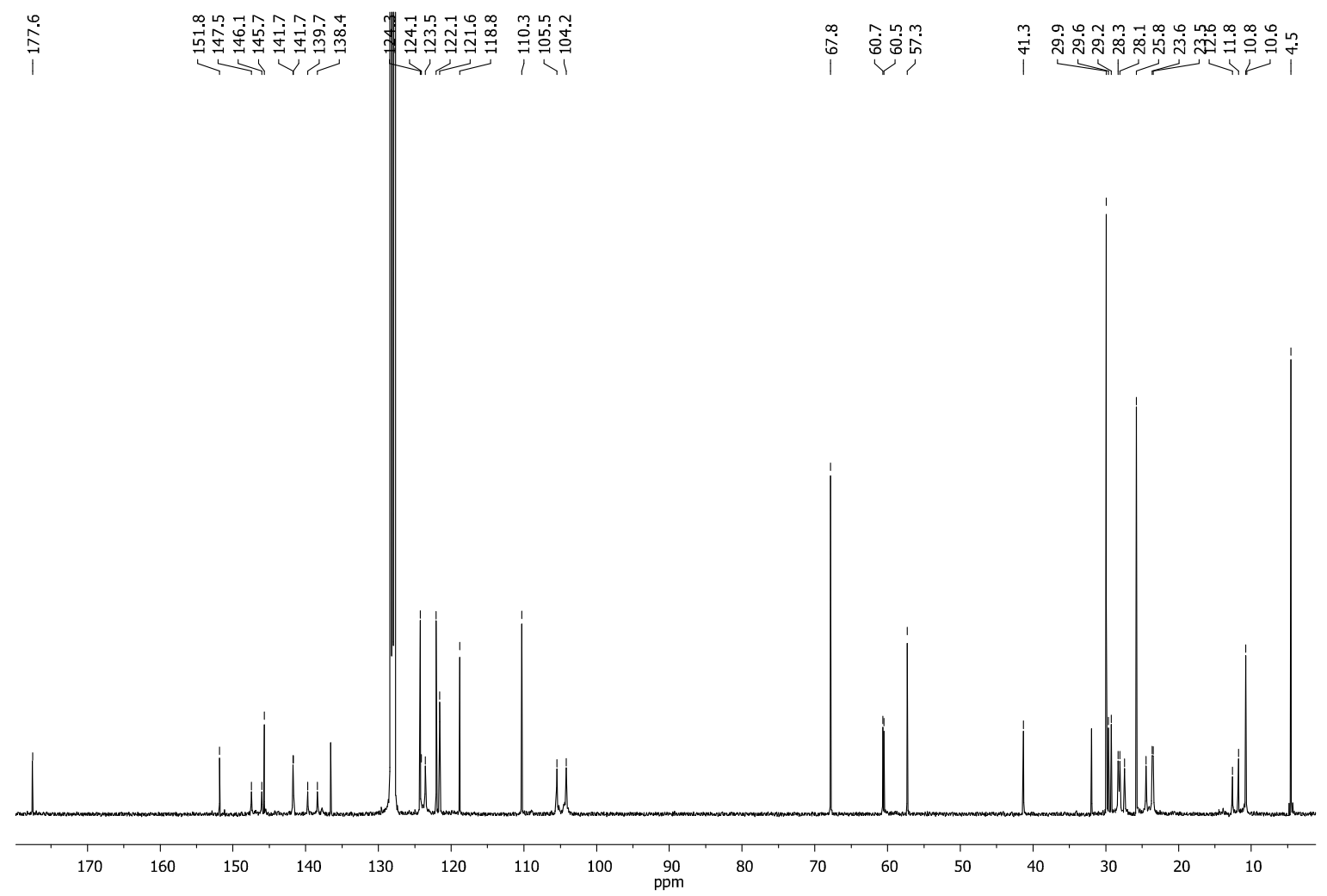

Figure S8. ${ }^{13} \mathrm{C}\left\{{ }^{1} \mathrm{H}\right\}$ NMR spectrum of $8\left(100 \mathrm{MHz}, \mathrm{C}_{6} \mathrm{D}_{6}, 293 \mathrm{~K}\right)$. 


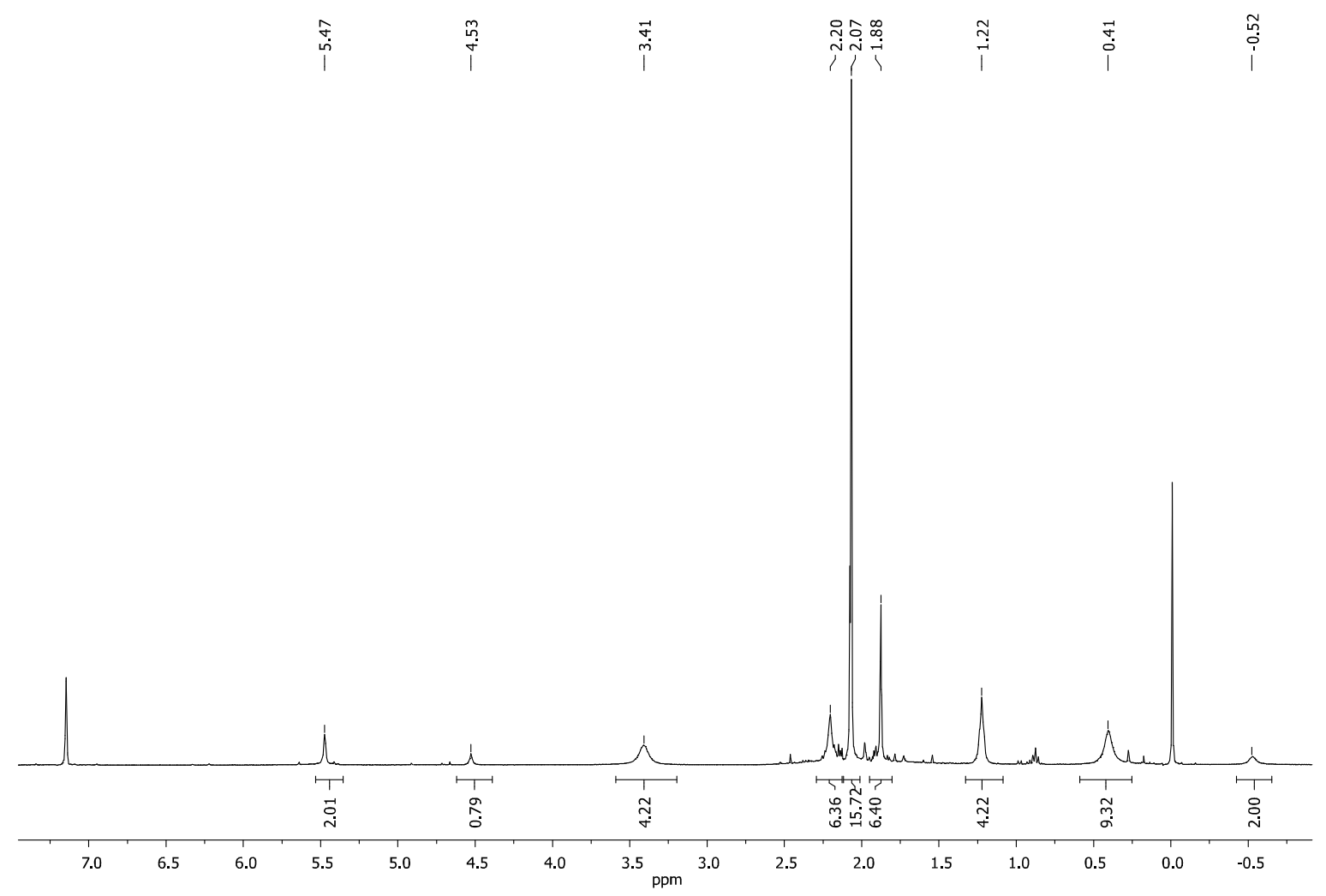

Figure S9. ${ }^{1} \mathrm{H}$ NMR spectrum of $9\left(400 \mathrm{MHz}, \mathrm{C}_{6} \mathrm{D}_{6}, 293 \mathrm{~K}\right)$.

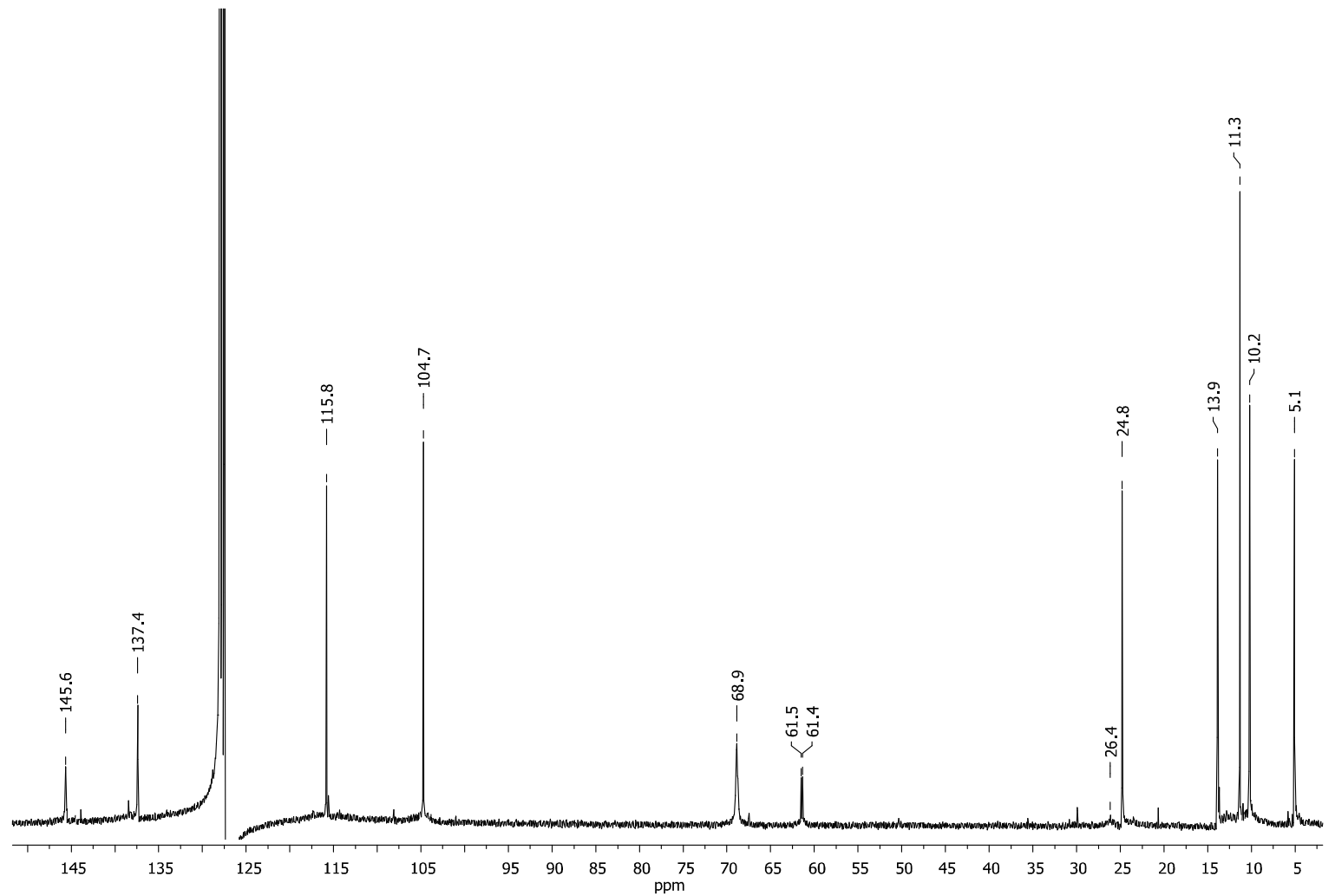

Figure S10. ${ }^{13} \mathrm{C}\left\{{ }^{1} \mathrm{H}\right\}$ NMR spectrum of $9\left(100 \mathrm{MHz}, \mathrm{C}_{6} \mathrm{D}_{6}, 293 \mathrm{~K}\right)$. 


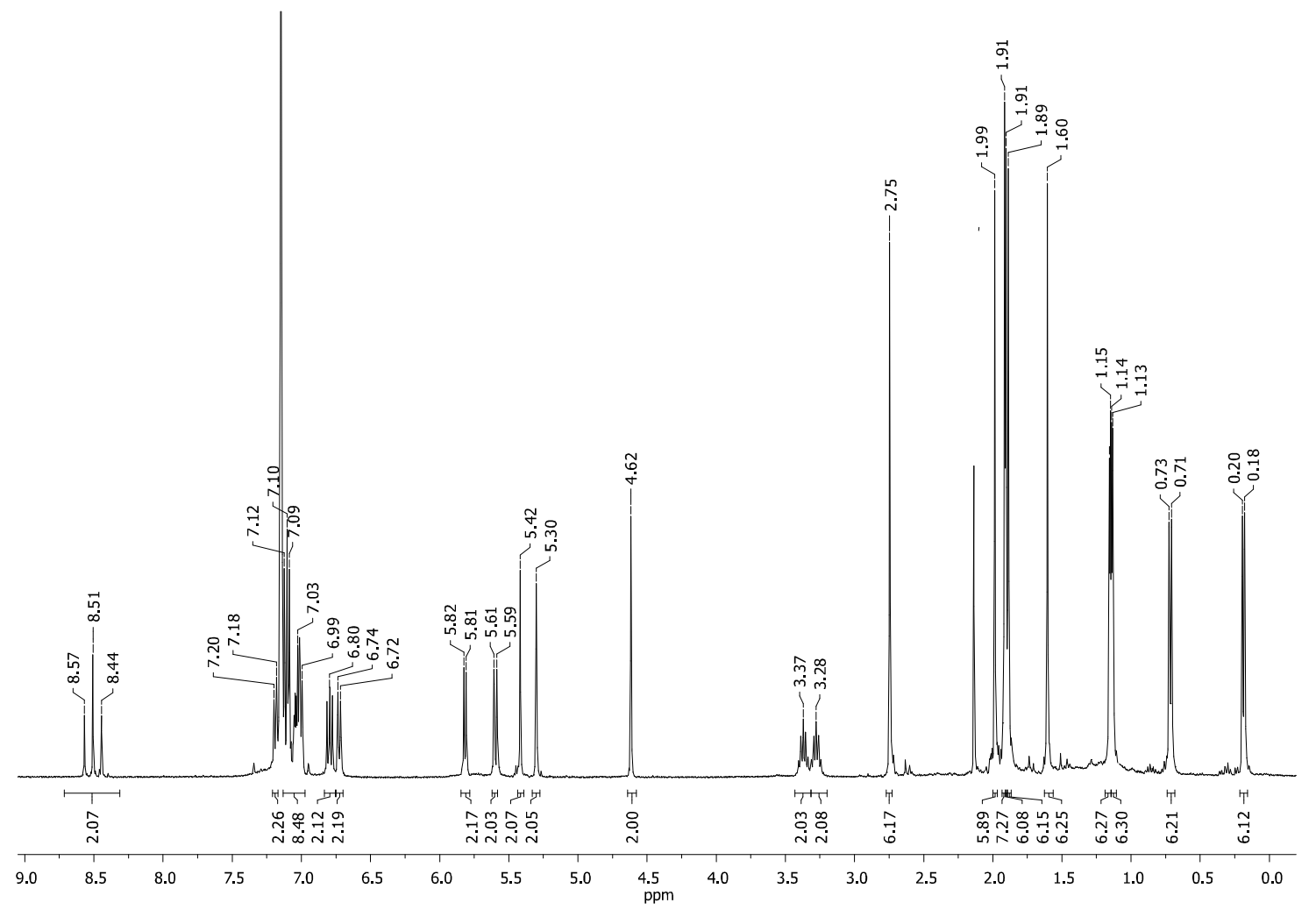

Figure S11. ${ }^{1} \mathrm{H}$ NMR spectrum of $\mathbf{1 0}\left(400 \mathrm{MHz}, \mathrm{C}_{6} \mathrm{D}_{6}, 293 \mathrm{~K}\right)$.

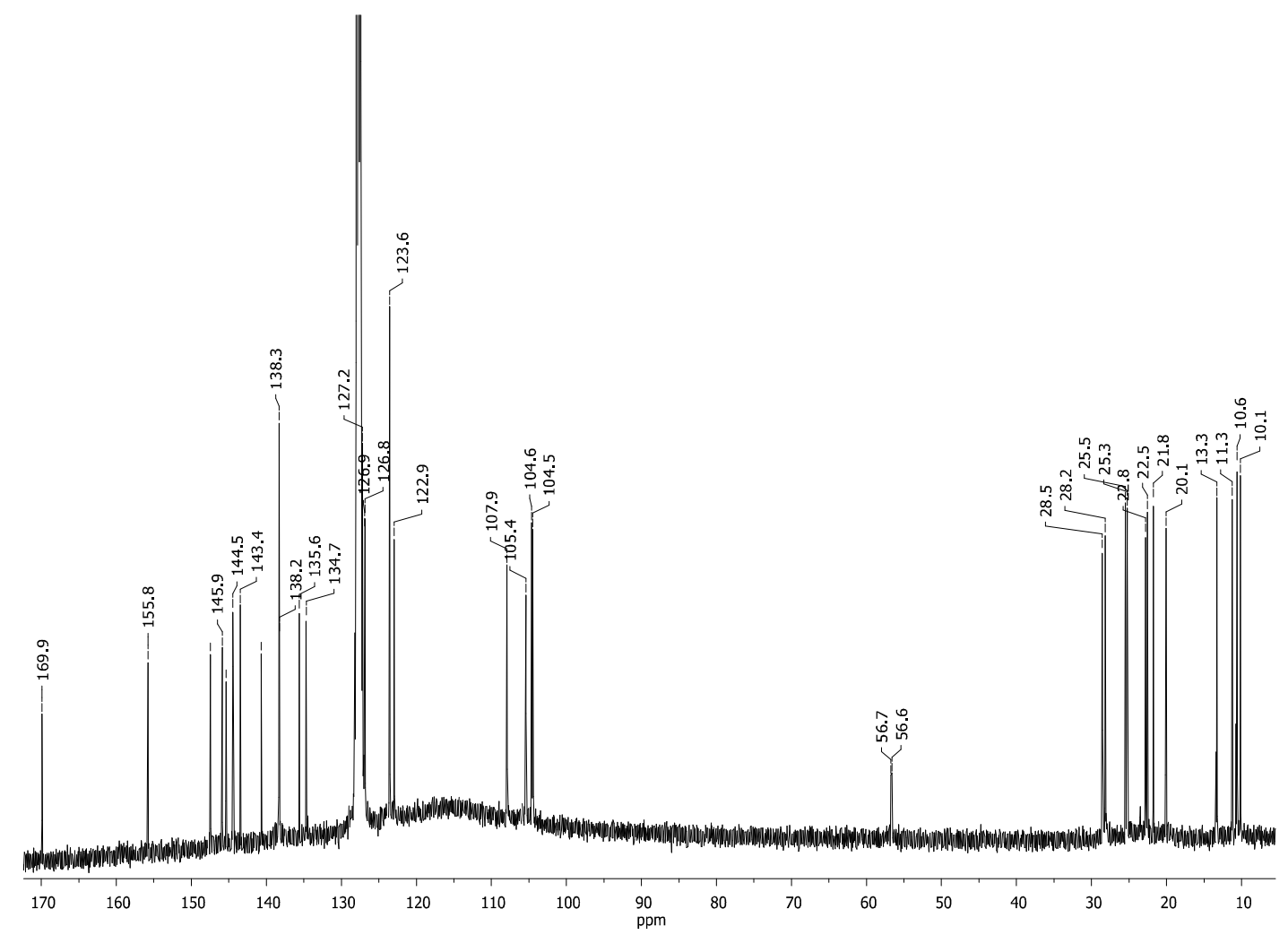

Figure S12. ${ }^{13} \mathrm{C}\left\{{ }^{1} \mathrm{H}\right\}$ NMR spectrum of $10\left(100 \mathrm{MHz}, \mathrm{C}_{6} \mathrm{D}_{6}, 293 \mathrm{~K}\right)$. 


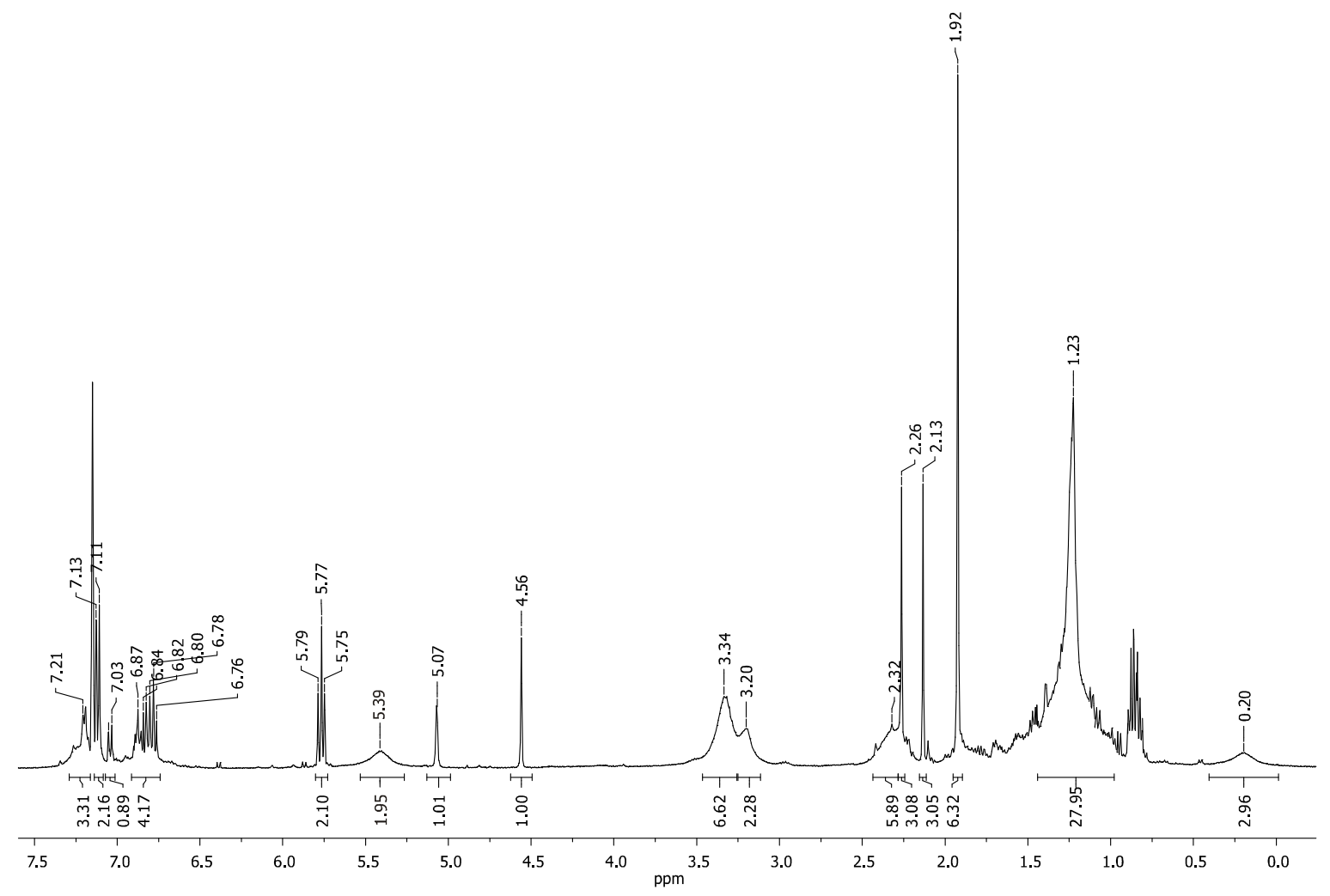

Figure S13. ${ }^{1} \mathrm{H}$ NMR spectrum of $11\left(400 \mathrm{MHz}, \mathrm{C}_{6} \mathrm{D}_{6}, 293 \mathrm{~K}\right)$.

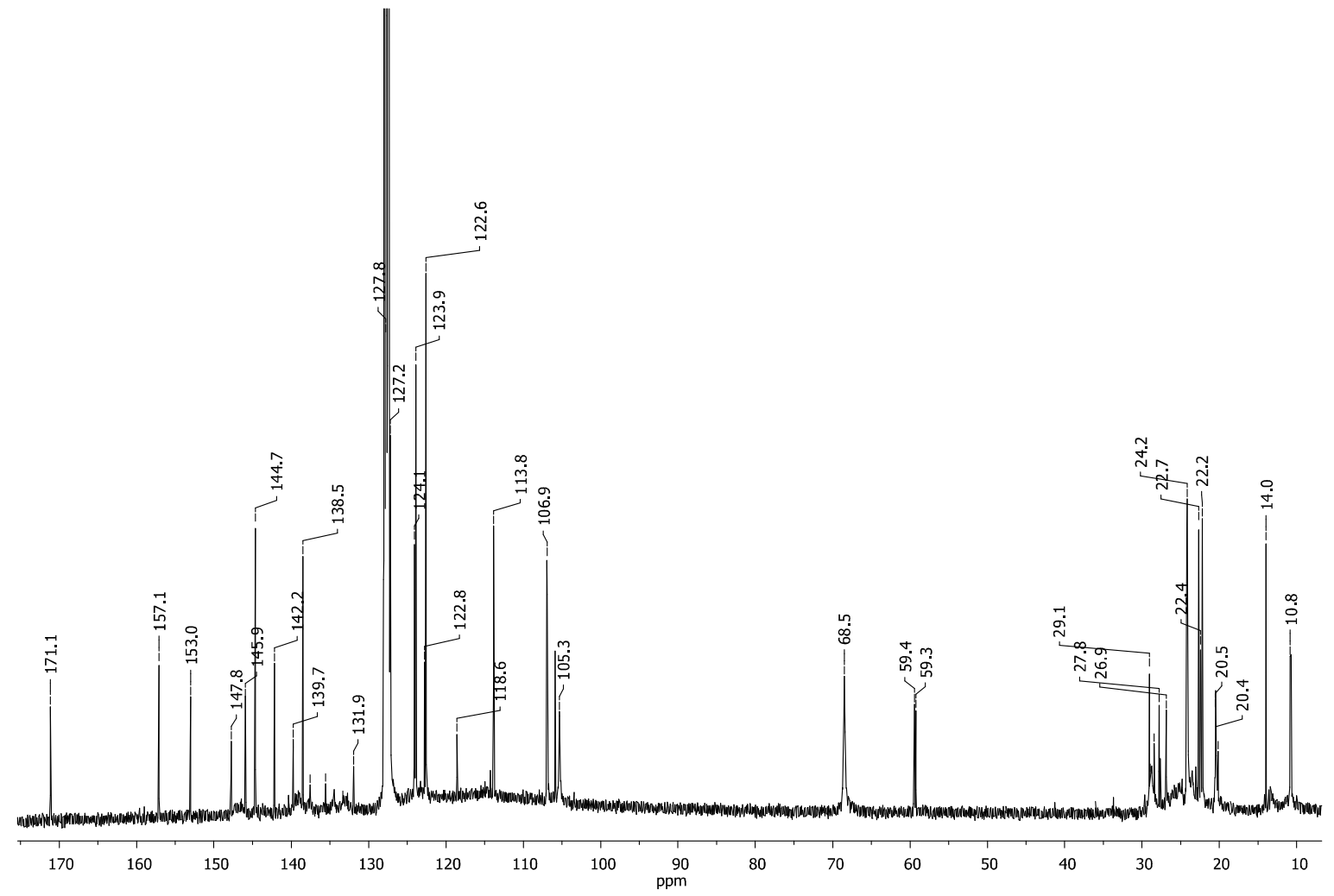

Figure S14. ${ }^{13} \mathrm{C}\left\{{ }^{1} \mathrm{H}\right\}$ NMR spectrum of $11\left(100 \mathrm{MHz}, \mathrm{C}_{6} \mathrm{D}_{6}, 293 \mathrm{~K}\right)$. 